\title{
Septicaemia and abdominal distension
}

\author{
Stephanie Farrand, Kalman Kafetz
}

A 79-year-old woman was admitted with tachycardia, pyrexia and hypotension. She had a raised white cell count of $25.7 \times 10^{9} / 1$ and deranged liver function tests. Her electrolytes, chest X-ray and abdominal X-ray were normal. Initial management was with broad-spectrum antibiotics. Blood cultures revealed staphylococci sensitive to flucloxacillin.

Lumbar spine X-ray, bone scan, abdominal ultrasound and knee aspiration were performed; degenerative changes of the lumbar spine were the only positive finding.

She remained apyrexial and her white count returned to normal, but her abdomen became progressively distended with sparse bowel sounds and mucus only passed per rectum. Abdominal $\mathrm{X}$-ray showed grossly distended loops of large bowel (figure 1). A barium enema was performed (figure 2).

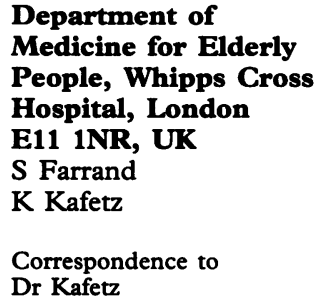

Accepted 25 September 1995
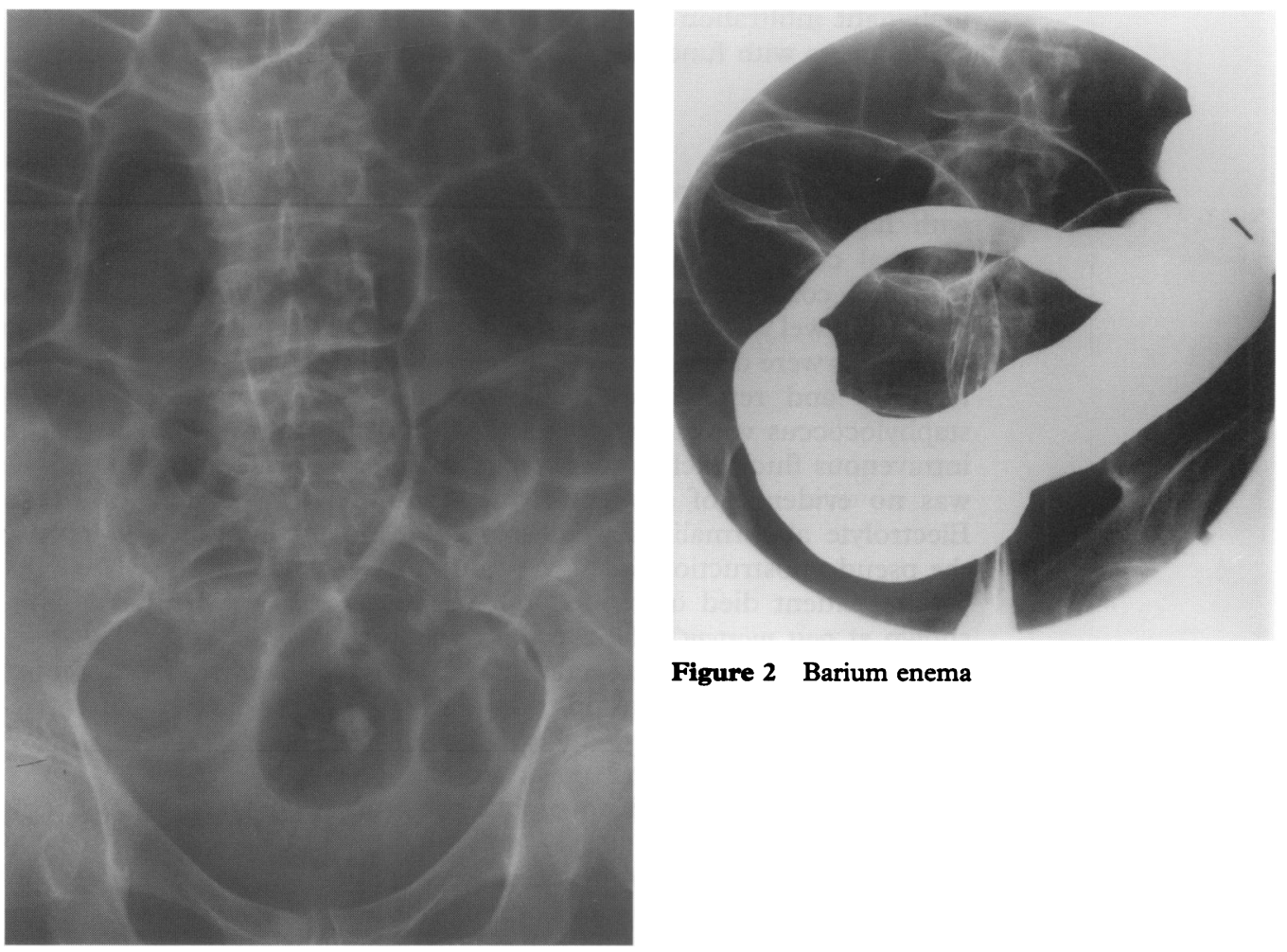

Figure 2 Barium enema

Figure 1 Abdominal X-ray

\section{Questions}

1 What complication of staphylococcal septicaemia has occurred?

2 Name four other factors that are associated with the development of this condition. 


\section{Answers}

QUESTION

She had developed acute colonic pseudoobstruction as illustrated by grossly distended loops of bowel on plain abdominal X-ray, and the barium enema showing moderate dilatation of the colon with a proximal segment of the sigmoid colon which is uniformly narrowed causing a partial hold-up of barium. Acute colonic pseudo-obstruction is a clinical condition with features suggesting large bowel obstruction but without a mechanical cause.

\section{QUESTION 2}

Multiple factors are known to be linked with the development of acute colonic pseudoobstruction (see box). The pathophysiology has not been elucidated. The most likely cause is ileus of a segment of bowel secondary to an imbalance of autonomic innervation. ${ }^{1}$ It was first described in 1948 by Ogilvie $^{2}$ who found malignant infiltration of the coeliac plexus in two patients with functional obstruction.

\section{Comment}

Despite apparent resolution of the septicaemia with high-dose antibiotics, the patient's abdominal condition did not resolve. She was managed conservatively with the expectation that her bowel would recover. After four weeks, antibiotics were discontinued. The septicaemia recurred and repeat blood cultures grew a staphylococcus with identical sensitivities and intravenous flucloxacillin was restarted. There was no evidence of any endocrine disorder. Electrolyte abnormalities were corrected, but the pseudo-obstruction failed to resolve.

The patient died of bronchopneumonia as shown at post mortem. The caecum, ascending colon and part of the transverse colon showed moderate dilatation, beyond this the colon appeared normal until there was distension again in the sigmoid and pelvic colon. The mucosa was normal and no organic obstruction was seen.

The body of L5 was soft with pus exuding from the periostium on the anterior surface with a tract of pus extending behind the psoas

1 Dorudi S, Berry AR, Kettlewell MWG. Acute colonic pseudo-obstruction. Br f Surg 1992; 79: 99-103.

2 Ogilvie $H$. Large intestine colic due to sympathetic deprivation. A new clinical syndrome. BMF 1948; 2: $671-3$

3 Davies DV, ed. Gray's anatomy. London: Longmans, 1967.
Factors linked with the development of pseudo-obstruction

- metabolic disturbance: hypokalaemia, hyponatraemia, hypocalcaemia, hypomagnesaemia

- systemic illness: malignancy, septicaemia, connective tissue disorders, systemic amyloid

- drugs: narcotics, calcium channel blockers, H2 blockers, tricyclic antidepressants, phenothiazines

- neurological disorders: muscular dystrophy, Parkinson's disease

- endocrine disorders: diabetes mellitus, hypothyroidism muscle on the right for several centimetres. $\vec{O}$ The superior hypogastric plexus lies anterior to $\exists$ L5. ${ }^{3}$ It is likely that pus affected this plexus ${ }_{\stackrel{\omega}{\omega}}$ resulting in an imbalance between the para- 80 sympathetic and sympathetic innervation caus- 3 ing unopposed action of the sympathetic system on either side of the narrowed portion of bowel with normal activity of the parasym- $\stackrel{G}{-}$ pathetic system around the narrowed (normal) ${ }^{\circ}$
segment.

The treatment options for acute colonic 9 pseudo-obstruction are conservative decom- $\vec{\circ}$ pression, correction of electrolyte abnormal- $\mathbb{D}$ ities, and treatment of any associated ${ }_{\mathbb{\Phi}}$ conditions or infection, pharmacological manipulation, ${ }^{4,5}$ colonoscopic decompression, ${ }^{1} \stackrel{\oplus}{\rightarrow}$ and, should these manoeuvres fail, surgery. ${ }^{1}$

This case demonstrates the difficulty of $\%$ conservative management in a patient considered unfit for surgical intervention, and the importance of the early recognition of this condition and its aetiology.

\section{Final diagnosis}

Staphylococcal septicaemia arising from osteomyelitis of L5. Acute colonic pseudo-obstruction.

Keywords: septicaemia, osteomyelitis, colonic pseudo-obstruction

We thank Dr Kathleen Thomas for the post mortem.

4 Neely J, Cathchpole B. Ileus: the restoration of alimentary tract motility by pharmacological means. Br $\mathcal{F}$ Surg 1971; 58:

5 MacColl C, MacCannell K, Baylis B, Lee S. Treatment of $N$ acute colonic pseudo-obstruction (Ogilvie's syndrome) with cisapride. Gastroenterology 1990; 98: 773-6. 